\title{
Effect of an anti-reflux medical device in the control of deflation cough: A placebo-controlled comparative study with an antacid drug in chronic coughers
}

\author{
Elisa Chellini, Federico Lavorini, Giacomo Campi, Claudia Mannini, Giovanni A. Fontana* \\ Department of Experimental and Clinical Medicine, University of Florence, Florence, Italy
}

\section{A R T I C L E I N F O}

Article history:

Received 3 February 2015

Received in revised form 4 May 2015

Accepted 6 May 2015

Available online 15 May 2015

\section{Keywords:}

Deflation cough

Chronic cough

Magnesium alginate

Simethicone

Aluminium hydroxide

Magnesium hydroxide

Reflux

\begin{abstract}
A B S T R A C T
Background: Deflation cough (DC), i.e. the cough-like expiratory expulsive efforts evoked by maximal lung emptying, is partially inhibited by prior intake of an antacid. We wished to compare the effects of an anti-reflux medical device (Gastrotuss ${ }^{\circledR}$ ) and of a widely used antacid drug (Maalox ${ }^{\circledR}$ ) on the number of expiratory thrusts evoked by maximal lung emptying in chronic cough patients.

Methods: Twenty consecutive chronic cough outpatients also presenting DC attended the clinic on three separate occasions and were requested to inhale to near total lung capacity and then exhale maximally for at least $6 \mathrm{~s}$. Trained investigators detected aurally the number of cough efforts evoked by maximal lung emptying prior to and 1, 5,10,30 e 60 min after administration of either Maalox ${ }^{\circledR}$, or Gastrotuss ${ }^{\circledR}$ or placebo. The liking of the administered agents was also rated.

Results: In control conditions, maximal lung emptying was consistently accompanied by the appearance of DC. The number of efforts was unchanged after placebo whereas it was markedly $(P<0.001)$ reduced 1 -10 min following Maalox ${ }^{\circledR}$ and Gastrotuss ${ }^{\circledR}$ administration. The value of liking for Gastrotuss ${ }^{\circledR}$ was greater $(\mathrm{P}<0.01)$ than those of Maalox ${ }^{\mathbb{B}}$ and placebo.

Conclusions: Pre-treatment with anti-reflux agents with a substantially different composition are equally effective in inhibiting DC. The liking of the two compounds used in the present experiments differed considerably and may be important to improve adherence to treatment in patients undergoing long-term therapy for reflux-related symptoms.
\end{abstract}

๑) 2015 Published by Elsevier Ltd.

\section{Introduction}

Recently, we have observed that during maximal lung emptying some patients with chronic cough and symptoms of gastrooesophageal reflux produce one or more cough efforts that typically occur when lung volume is emptied to near residual volume $[1,2]$. The mechanism underlying this phenomenon, termed "deflation cough" (DC), appears to depend upon oesophageal acidification, since in the majority of patients DC is markedly reduced or even abolished by pre-treatment with a 40-ml Maalox ${ }^{\circledR}$ (Novartis International AG, Basel, $\mathrm{CH}$ ), a solution containing aluminium hydroxide $(3.25 \mathrm{~g} / 100 \mathrm{ml})$ and magnesium hydroxide

\footnotetext{
Abbreviations: DC, deflation cough; GOR, gastro-oesophageal reflux.

* Corresponding author. Department of Experimental and Clinical Medicine, Largo Brambilla, 3, 50134 Firenze, Italy. Tel.: +39 055 7947516; fax: +39 055 4223202.

E-mail address: giovanni.fontana@unifi.it (G.A. Fontana).
}

(3.65 g/100 ml) [1]. The hydroxides react with excess acid in the stomach, reducing its acidity [3,4]; the agent is commonly prescribed as an antacid to minimise the clinical symptoms of oesophageal acidification in patients with gastro-oesophageal reflux (GOR) [5] and its inhibitory action on DC was shown to be significantly stronger than that of placebo [1]. Gastrotuss ${ }^{\circledR}$ (Drugs Mineral and Generics, Pomezia, Rome, I) is a liquid preparation, registered as a medical device, largely employed in the control symptoms of GOR, including chronic cough [6]. The device is an association of different agents including simethicon - an anti-foaming agent that decreases the surface tension of gas bubbles - and magnesium alginate, i.e. the magnesium salt of alginic acid which is administered orally in the treatment of GOR [6]. After ingestion, the device combines with gastric acid to form a viscous gel, which floats on top of the gastric contents and acts as a physical barrier to reflux [6]. However, the effectiveness of Gastrotuss ${ }^{\circledR}$ in the control of DC has not been assessed. Thus, the primary objective of the study was to assess the effectiveness of Gastrotuss ${ }^{\mathbb{R}}$ in preventing or reducing DC 
in a group of outpatients with chronic cough. The secondary objective of the study was to compare the effectiveness of Maalox ${ }^{\mathbb{R}}$ and Gastrotuss ${ }^{\circledR}$ in the control of DC and to assess whether patients show any preferences regarding the liking of the agents employed.

\section{Materials and methods}

\subsection{Patients}

We recruited 20 consecutive outpatients (13 females, Table 1) with chronic cough of any origin and DC who attended the Cough Centre at the Azienda Ospedaliero Universitaria Careggi, Florence, Italy. Patients were non-smokers or ex-smokers $(n=6)$ for longer than 24 months, none of them reported recent ( $<4$ weeks) airway infections. Patients were requested to be off any acid-suppressing therapy for at least 1 week before enrollment in the study. All procedures were in accordance with the Helsinki Declaration and the study was approved by the local Institutional Review Board (n. 869/13); patients gave their signed informed consent to participate.

\subsection{Treatments}

Maalox $^{\circledR}(40 \mathrm{ml})$, or Gastrotuss ${ }^{\circledR}(40 \mathrm{ml})$ or placebo, i.e. a solution freshly prepared with $30 \mathrm{ml}$ mineral water, $10 \mathrm{ml}$ UHT milk and 10 drops of a multivitamin compound [1], were employed. A single dose of the active agents or placebo was randomly administered at each scheduled visit. The sequence of administered treatments was allocated according to an on-line randomization web site (www. randomization.com).

\subsection{Study design and protocol}

This was a randomized, placebo-controlled, double blind, crossover study. Blindness was guaranteed by the fact that all treatments were administered by indistinguishable syringes into the patient's mouth, the identity of which were blinded to patients and investigators. Patients attended the clinic on three occasions separated by a time interval of $48-72 \mathrm{~h}$. Food intake was withheld $3 \mathrm{~h}$ prior to each study day. At the clinic, patients underwent a general clinical assessment and the assessment of DC as described in the literature [1]. In brief, patients inhaled to near total lung capacity and then exhaled slowly down to near residual volume. During the manoeuvre patients wore a nose clip and breathed freely through a mouthpiece to prevent pursing of the lips, a phenomenon that in our experience may affect the appearance of DC. Patients were trained to exhale maximally as in an attempt at emptying out the lung as much as possible for at least $6 \mathrm{~s}$. Trained investigators detected aurally the number of cough efforts evoked by maximal lung emptying prior to (i. e., at baseline) and 1, 5, 10, 30 e $60 \mathrm{~min}$ after each treatment and noted for subsequent analyses. At baseline, the manoeuvre was repeated 3-5 times, and a 5 min recovery period was allowed between each expiratory effort. After completion of each trial, patients were requested to rate the liking of the administered agent according to a method described previously [7]. In brief, patients rated the taste using a $10 \mathrm{~cm}$ long visual analogue scale. The extremes of the scale were classified from 0 (extremely poor) to 10 (excellent).

\subsection{Data analysis}

Based on the results of a previous investigation [1], the sample size was chosen to design the study to have a $80 \%$ statistical power of detecting a $50 \%$ reduction in baseline DC frequency with the use of one-way repeated measure analysis of variance (ANOVA) and a significance level of 0.05 . All expulsive efforts recorded during each maximal expiration were considered. Deflation cough frequency was taken as the number of expiratory efforts recorded during each maximal expiration. The number of DC events recorded at baseline was pooled and averaged for subsequent calculations. Comparisons between DC frequencies recorded at baseline and after placebo, Maalox $^{\circledR}$, and Gastrotuss ${ }^{\circledR}$ administration were performed by twoway, nonparametric, repeated-measure ANOVA followed by posthoc tests. Treatments (i. e. Gastrotuss ${ }^{\circledR}$, Maalox $^{\circledR}$ and placebo) and the time intervals after administration of each drug were factors in the ANOVA. This statistical analysis allowed us to investigate, for each subject, interactions between drugs and the time course after their administration. Ratings of anti-reflux agents' taste obtained at the end of each trial were compared by one-way ANOVA followed by Dunn's multiple comparisons test. All reported values are means \pm standard deviations (SD); a P value $<0.05$ was taken as significant.

Table 1

Patients' anthropometric, functional and clinical characteristics.

\begin{tabular}{|c|c|c|c|c|c|c|c|}
\hline Pt. no. & Sex & Age (years) & BMI & $\mathrm{FEV}_{1} / \mathrm{FVC}$ & Cough duration (months) & Most prominent associated symptoms & Ongoing treatment \\
\hline 1 & M & 55 & 29.07 & 80 & 72 & Regurgitation & PPI \\
\hline 2 & $\mathrm{~F}$ & 59 & 21.36 & 75 & 96 & Dyspnoea, dysphonia, heartburn regurgitation & LAMA, PPI \\
\hline 3 & $\mathrm{~F}$ & 51 & 20.81 & 79 & 12 & Dysphonia, indigestion & None \\
\hline 4 & M & 23 & 19.59 & 77 & 24 & Heartburn, indigestion & None \\
\hline 5 & $\mathrm{~F}$ & 57 & 25.40 & 79 & 12 & Dysphonia, regurgitation, thoracic pain & None \\
\hline 6 & M & 67 & 31.25 & 81 & 11 & Dysphonia, indigestion & None \\
\hline 7 & $\mathrm{~F}$ & 40 & 22.59 & 73 & 60 & Dyspnoea, indigestion, regurgitation & PPI \\
\hline 8 & $\mathrm{~F}$ & 70 & 25.39 & 79 & 24 & Dysphonia, dyspnoea, regurgitation & ICS, PPI \\
\hline 9 & $\mathrm{~F}$ & 66 & 23.83 & 77 & 10 & Dysphonia, heartburn & PPI \\
\hline 10 & M & 62 & 25.26 & 72 & 36 & Heartburn, indigestion, regurgitation & None \\
\hline 11 & M & 37 & 24.38 & 79 & 9 & Dysphonia, heartburn & PPI \\
\hline 12 & $\mathrm{~F}$ & 67 & 29.64 & 77 & 10 & Dysphonia dyspnea, indigestion & None \\
\hline 13 & M & 55 & 25.10 & 86 & 8 & Dysphonia, heartburn & None \\
\hline 14 & $\mathrm{~F}$ & 29 & 24.50 & 80 & 9 & Heartburn, regurgitation & None \\
\hline 15 & $\mathrm{~F}$ & 61 & 27.18 & 81 & 12 & Dyspnoea, heartburn & LAMA, PPI \\
\hline 16 & M & 60 & 27.12 & 77 & 84 & Heartburn, regurgitation & None \\
\hline 17 & $\mathrm{~F}$ & 65 & 28.09 & 73 & 72 & Dysphonia, dyspnoea, regurgitation & PPI, LABA, ICS \\
\hline 18 & $\mathrm{~F}$ & 68 & 25.71 & 79 & 7 & Heartburn, thoracic pain & PPI \\
\hline 19 & $\mathrm{~F}$ & 50 & 25.10 & 81 & 100 & Heartburn, indigestion, regurgitation & PPI, prokinetics \\
\hline 20 & $\mathrm{~F}$ & 70 & 24.91 & 74 & 96 & Dyspnoea, heartburn, thoracic pain & ICS, LABA, PPI \\
\hline Mean & - & 55.60 & 26.79 & 77.95 & 38.20 & & \\
\hline SD & & 13.65 & 3.23 & 3.39 & 35.35 & & \\
\hline
\end{tabular}

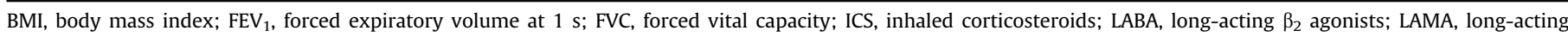
muscarinic antagonist; Pt, patient; PPI, proton pump inhibitors. 


\section{Results}

In control conditions, maximal lung emptying was consistently accompanied by the appearance of DC in all patients examined. The time course of mean changes in DC frequency following administration of all study agents is depicted in Fig. 1 . The mean $\left({ }_{ \pm} \mathrm{SD}\right)$ number of deflation coughs recorded in control condition was $6.35 \pm 1.79$. This value was unchanged $1 \mathrm{~min}$ following placebo $(6.00 \pm 1.24)$, whereas it was markedly $(\mathrm{P}<0.001)$ reduced to $3.4 \pm 1.29$ and $2.07 \pm 1.411$ min following Maalox ${ }^{\circledR}$ and Gastrotuss ${ }^{\circledR}$ administration, respectively. It should be noted that placebo, although ineffective in the patients as a group, actually reduced DC frequency in four of them. It was, however, completely ineffective in 16 patients. Conversely, 1 min after Maalox ${ }^{\circledR}$ administration, DC was abolished in 13 patients and its frequency was reduced in 7 of them. Following Gastrotuss ${ }^{\circledR}$, DC was completely abolished in 12 patients and its frequency was reduced in 8 . The reduction in DC frequency persisted for as long as $10 \mathrm{~min}$ with both Maalox ${ }^{\circledR}$ and Gastrotuss $^{\circledR}$; as a rule, values of DC frequency resumed their control value 30 min following anti-reflux agents administration (Fig. 1). Although the reduction in mean DC frequency after Maalox ${ }^{\mathbb{B}}$, and Gastrotuss $^{\circledR}$ was significantly greater than with placebo ( $\mathrm{P}$ always $<0.001$ ), no difference was found between the magnitude of mean DC inhibition with Maalox ${ }^{\circledR}$ and that with Gastrotuss ${ }^{\circledR}$. Patients in whom DC was only partially inhibited by the active agents had clinical characteristics similar to those in whom antacid administration was fully effective in abolishing deflation cough. As a rule, patients who displayed a weaker DC inhibition to one of the active agents, also failed to respond fully to the other (data not shown).

As shown in Fig. 2, the mean $( \pm S D)$ value of liking was $5.73 \pm 1.48$ for Gastrotuss ${ }^{\circledR}, 3.82 \pm 1.48$ for Maalox ${ }^{\circledR}$, and $4.55 \pm 1.60$ for placebo. Between group analysis demonstrated higher

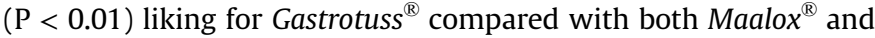
placebo, whereas the latter two agents reached comparable liking scores.

\section{Discussion}

The present findings firstly confirm our previous observation that, in patients with chronic cough and DC, pre-treatment with high dose of an antacid drug or a reflux-preventing medical device significantly inhibits DC [1]. Secondly, they also confirm that DC inhibition by these agents is of variable degree, ranging from complete abolition of DC in some patients to a slight DC reduction

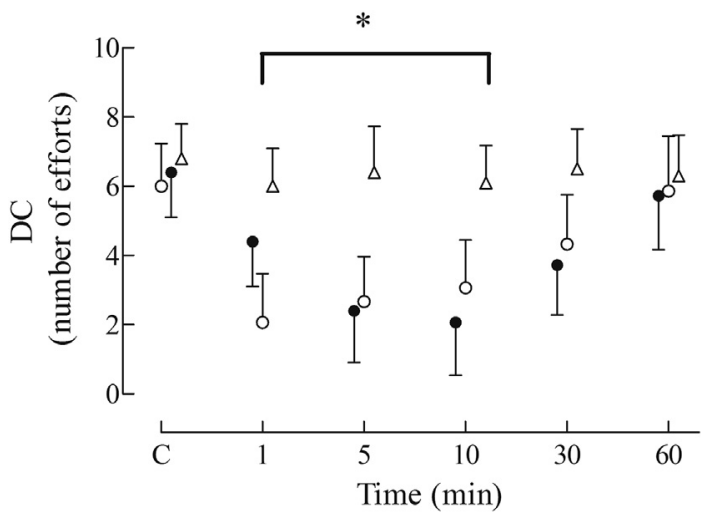

Fig. 1. Time course of mean (SD) changes in deflation cough (DC) frequencies observed following administration of Gastrotuss ${ }^{\circledR}$ (empty circles) Maalox ${ }^{\circledR}$ (filled circles), or placebo (triangles). $\mathrm{C}$, control condition. The asterisks indicate the time points at which we detected a significant $(\mathrm{P}<0.01)$ difference in DC frequency between Maalox ${ }^{\circledR}$ and Gastrotuss $^{\circledR}$ compared to placebo.

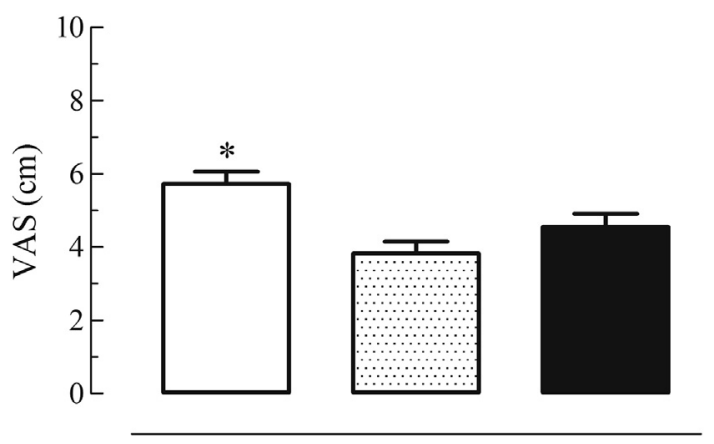

Fig. 2. Mean (SD) scores of visual analogue scale (VAS) rating the liking of Gastrotuss ${ }^{\circledR}$ (empty column), Maalox ${ }^{\circledR}$ (grey column) or placebo (black column); ${ }^{*}, \mathrm{P}<0.01$ for Gastrotuss ${ }^{\circledR}$ compared to the other agents.

in others [1]. Third, the results demonstrate for the first time that an anti-reflux medical device or an antacid drug with a substantially different composition and mechanism of action are equally effective in inhibiting DC. Last, but not least, the results also show that the liking of the two compounds used in the present experiments differs considerably.

We have enrolled patients with chronic cough of any origin and DC as assessed at the first clinical evaluation. Since the primary purpose of the study was to evaluate the effectiveness of an antireflux medical device or an antacid drugs in controlling the DC, we were not interested in any additional recruitment criterion nor in performing a full clinical workup to establish a diagnosis of the chronic cough at the time of the first visit. However, since DC has been shown to be inhibited by high dose of antacid pre-treatment in both the present and in previous experiments [1], it can be inferred that the pathophysiological mechanisms responsible for chronic cough in patients examined here likely included GOR.

The pathophysiology of DC remains to be fully elucidated $[1,2]$. Although the chemical composition of the two products studied varies considerably (see above), their effectiveness in controlling DC appears to be remarkably similar, suggesting comparable protective effect against the stimuli that are involved in DC mediation at the oesophageal level. A detailed discussion on the mechanisms by which these products prevent DC would be beyond the scope of this study. Both Maalox ${ }^{\circledR}$ and Gastrotuss ${ }^{\circledR}$ have been shown to reduce on acid reflux and total acid exposure due to local chemical $\mathrm{H}^{+}$- and pepsin-binding capacity [4]; in addition, due to the presence of simethicone, Gastrotuss ${ }^{\mathbb{R}}$ may also improve gastric distension with synergistic reduction of transient lower oesophageal sphincter relaxation [6]. Since both of them seem to counteract the effects of gastric refluxate on the oesophageal mucosa [3], it seems plausible to infer that the mechanism of DC inhibition by these agents involves the blocking of reflex responses of oesophageal origin.

Most over-the-counter antitussive liquid preparations are sweet and viscous due to added sweeteners, such as glucose, sucrose and honey [8]. Sweeteners are usually listed as excipients, presumably used for flavour; they impart a pleasant taste and also could mask unpleasant tastes of other ingredients, because sweetness tends to suppress bitterness [8]. Interestingly, sweetened preparations have significant antitussive effects even when they contain no drugs [9].The fact that honey has been used for thousands of years as a cough remedy is consistent with this idea. It is unclear whether sweetness per se is responsible for the relief that sweet preparations provide, but one study has shown that rinsing the mouth with sucrose and inhaling menthol vapour significantly increased cough thresholds to capsaicin [10]. Both Maalox ${ }^{\mathbb{B}}$ and Gastrotuss ${ }^{\mathbb{B}}$ contain sweeteners and flavourings as excipients; therefore we cannot rule 
out that these ingredients may have a role in the DC inhibition observed in the present study. Further studies are required to investigate the effects of sweeteners preparation lacking of pharmacologically active ingredients on the genesis of DC.

Although the role of oesophageal acidification appears to be prominent in most instances, it seems worth recalling that in some patients the DC is only slightly diminished by prior intake of an antacid [1]. Of note, patients who displayed a weak response to one of the products, also did so in response to the other one, suggesting that failure to markedly suppress the DC is independent of the type or composition of the agent, but rather points to the possibility that additional mechanisms other than oesophageal acidification are implicated in genesis of DC in these patients. In this connection, it has been proposed [2] that the DC represents a positive feed-back reflex response also implicating sensory nerve terminals and afferent fibres in the lungs that are activated by the changes in intra-thoracic caused by maximal lung emptying [11]. With DC, a voluntary maximal deflation of the lungs to residual volume augments (paradoxically) the existing expiratory drive with further brief expiratory efforts (the DC) [2]. Additional investigations are required to further clarify the role of sensory signals of pulmonary origin in the mediation of DC.

Various liquid anti-reflux or antacid agents such as those employed here are available both as over-the-counter or prescription medications for the treatment of GOR, peptic ulcer disease and other ill-defined causes of dyspepsia [12]. According to published guidelines [13], antacids or anti-reflux products are also widely employed in the control of GOR symptoms, including chronic chough. A possible drawback of antacid therapy is the need of frequents drug administration, since the duration of action of these compounds is generally short lasting [14]. On the other hand, the need of frequents administration is known to negatively impact on patient compliance to the therapy, especially when the liking of the compound is low. A patient may be more likely to choose an antireflux treatment if it is effective and palatable, particularly if the taste of the agent is pleasant [7]. We have shown that, when taken over short period of time to control DC in our experimental conditions, the liking of Gastrotuss ${ }^{\circledR}$ was significantly higher than that of Maalox ${ }^{\circledR}$. In consequence, it seems logical to assume that a highly palatable anti-reflux medical device such as Gastrotuss ${ }^{\circledR}$ may be advantageous in the long term treatment of patients with refluxrelated symptoms.

\section{Conclusions}

We have shown that DC can effectively be controlled by high dose of an anti-reflux medical device or an antacid drug, a finding that reinforces the role of oesophageal acidification in the genesis of DC. However, the results provide additional evidence that other mechanisms, most likely of bronchopulmonary origin also contribute, at least in a subset of patients. The organoleptic characteristics of anti-reflux/antacid products may be important to improve adherence to treatment in patients undergoing long-term therapy for reflux-related symptoms.

\section{Funding sources}

The research had no funding sources.

\section{Conflict of interests statement}

The Authors declare no conflict of interest related to this work.

\section{References}

[1] F. Lavorini, G.A. Fontana, E. Chellini, C. Magni, M. Pistolesi, J.G. Widdicombe, Respiratory expulsive efforts evoked by maximal lung emptying, Chest 140 (2010) 690-696.

[2] F. Lavorini, G. Fontana, E. Chellini, C. Magni, M. Pistolesi, J. Widdicombe, The Fontana paradoxical reflex? Chest 140 (2011) 586-588.

[3] P. Netzer, A. Brabetz-Hofliger, R. Brundler, B. Flogerzi, J. Husler, F. Halter, Comparison of the effect of the antacid Rennie versus low-dose H2-receptor antagonists (ranitidine, famotidine) on intragastric acidity, Aliment. Pharmacol. Ther. 12 (1998) 337-342.

[4] R.A. Faaij, J.M. Van Gerven, I. Jolivet-Landreau, A.A. Masclee, E.M. Vendrig, R.C. Schoemaker, L.D. Jacobs, A.F. Cohen, Onset of action during on-demand treatment with maalox suspension or low-dose ranitidine for heartburn, Aliment. Pharmacol. Ther. 13 (1999) 1605-1610.

[5] D.L. Decktor, M. Robinson, S. Gottlieb, Comparative effects of liquid antacids on Esophageal and gastric pH in patients with heartburn, Am. J. Ther. 2 (1995) $481-486$.

[6] D. Ummarino, E. Miele, M. Martinelli, E. Scarpato, F. Crocetto, E. Sciorio, A. Staiano, Effect of magnesium alginate plus simethicone on gastroesophageal reflux in infants, J. Pediatr. Gastroenterol. Nutr. 60 (2) (2015 Feb) 230-235.

[7] M.E. Temple, M.C. Nahata, Comparative palatability of 22 liquid antacids, Aliment. Pharmacol. Ther. 14 (2000) 421-425.

[8] P.M. Wise, P.A. Breslin, P. Dalton, Effect of taste sensation on cough reflex sensitivity, Lung 192 (2014) 9-13.

[9] R. Eclles, Mechanisms of the placebo effect of sweet cough syrups, Respir. Physiol. Neurobiol. 152 (2006) 340-348.

[10] P.M. Wise, P.A. Breslin, P. Dalton, Sweet taste and menthol increase cough reflex thresholds, Pulm. Pharmacol. Ther. 25 (2012) 236-241.

[11] J. Widdicombe, Airway receptors, Respir. Physiol. 125 (2001) 3-15.

[12] Antacids, in: B.R. Olin (Ed.), Drugs Facts and Comparisons, JB Lippincott Co, St Louis, 1999, pp. 291a-298a.

[13] G.N. Tytgat, K. McColl, J. Tack, G. Holtmann, R.H. Hunt, P. Malfertheiner A.P. Hungin, Batchelor HK.Aliment new algorithm for the treatment of gastrooesophageal reflux disease, Pharmacol. Ther. 27 (2008) 249-256.

[14] K.G. Mandel, B.P. Daggy, D.A. Brodie, H.I. Jacoby, Review article: alginate-raft formulations in the treatment of heartburn and acid reflux, Aliment. Pharmacol. Ther. 14 (2000) 669-690. 